\title{
SÍNTESE MECANOQUÍMICA DE AZINAS ACÍCLICAS SIMÉTRICAS E DETERMINAÇÃO DO FATOR DE PROTEÇÃO SOLAR UVB IN VITRO
}

Wesley Randson de Alcantara Campos ${ }^{\mathrm{a}}$, Débora Caroline Marques de Souzaa ${ }^{\mathrm{a}}$, Délis Galvão Guimarães ${ }^{\mathrm{a}}$, Victória Laysna dos Anjos Santos ${ }^{\mathrm{a}}$, Arlan de Assis Gonsalves ${ }^{\mathrm{a}}$ e Cleônia Roberta Melo Araújo ${ }^{*, \mathrm{a}, \odot}$

${ }^{a}$ Colegiado de Ciências Farmacêuticas, Universidade Federal do Vale do São Francisco, 56304-917 Petrolina - PE, Brasil

Recebido em 15/10/2018; aceito em 10/01/2019; publicado na web em 27/02/2019

\begin{abstract}
MECHANOCHEMICAL SYNTHESIS OF SYMMETRIC ACYCLIC AZINES AND DETERMINATION OF THE UVB SOLAR PROTECTION FACTOR in vitro. Skin cancer is a frequent neoplasm in Brazil and this, is observed the importance of the development of new sunscreens using simple methodologies capable of reducing production costs. In this context, six symmetric acyclic azines were synthesized and characterized, with reaction yields between 54 and 84\%, short reaction times (5 to $10 \mathrm{~min}$ ), and manual grinding as an energy source. Sunscreens $10 \%(\mathrm{~m} / \mathrm{m})$ of these compounds were produced for the in vitro determination of the solar protection factor UVB (SPF-UVB), of the the six formulations containing the synthesized azines, four showed higher photoprotective capacity in the UVB region (AZN-BZD, AZN-2OH, AZN-4OH and AZN-CIN), with SPF-UVB equivalent or higher than benzophenone-3 (BZF) and at the octylmethoxycinnamate (OMC), commercial chemical filters. The AZN-VAN and AZN-4DAB formulations presented high photoprotective capacity in the UVA region, resulting in lower SPF-UVB when compared to the other synthesized azines, but were still superior to the BZF and equivalent to the OMC. All the azines synthesized showed superior FPSUVB to their precursor aldehydes, evidencing the increase of the photoprotective capacity due to the insertion of the azine group.
\end{abstract}

Keywords: mechanosynthesis; grinding synthesis; sunscreen; chemical sunscreen.

\section{INTRODUÇÃo}

O Instituto Nacional do Câncer (INCA) estima que no Brasil o câncer de pele seja o mais frequente, correspondendo a $30 \%$ de todos os tumores malignos registrados. Classificados em dois tipos, melanoma e não melanoma, os tumores de pele do tipo melanoma representam apenas 3\% das neoplasias malignas deste órgão, embora seja considerado o tipo mais grave em razão da sua elevada probabilidade de metástase. Em 2013 foram registrados no país 3.316 mortes por câncer de pele segundo dados do Sistema de Informações sobre Mortalidade (SIM) e, para 2018, o INCA estima o surgimento de 171.840 novos casos. ${ }^{1}$

Os protetores solares de amplo espectro (UVA/UVB) são tidos como um dos mais importantes agentes preventivos ao câncer de pele, desde que usados adequadamente e de forma frequente. ${ }^{2}$ Segundo a Agência Nacional de Vigilância Sanitária (ANVISA), os protetores solares são definidos como preparações cosméticas que protegem a pele contra a radiação ultravioleta B (UVB - 290-320 nm) e A (UVA - 320-400 nm), absorvendo, dispersando ou refletindo a radiação. ${ }^{3}$

Apesar do seu extenso litoral e do clima predominantemente tropical, no Brasil, $64 \%$ das pessoas não fazem uso de fotoprotetores, de acordo com dados da última campanha de combate ao câncer de pele realizada pela Sociedade Brasileira de Dermatologia (SBD). ${ }^{4}$ Acredita-se que a baixa adesão ao uso diário de protetores solares pelos brasileiros está relacionada ao seu elevado custo, uma vez que a categorização dos mesmos como cosméticos gera o acréscimo de impostos dos quais os medicamentos são isentos, em especial os tributos de competência estadual, como por exemplo, o Imposto sobre Circulação de Mercadorias e Serviços (ICMS). ${ }^{5}$

A obtenção de fotoprotetores empregando metodologias sustentáveis e baseadas nos princípios da química verde, a exemplo da mecanoquímica, representa uma alternativa para redução dos custos de produção dos filtros solares. Portanto, a diminuição do uso de

*e-mail: cleonia.araujo@univasf.edu.br solventes, a síntese à temperatura e pressão ambientes, a menor geração de resíduos, além da possibilidade de dedução de tributos das empresas que aplicam ou têm uma postura de responsabilidade socioambiental, podem implicar em redução do preço final do produto.

Diante deste contexto, o presente trabalho descreve a síntese, caracterização e determinação do fator de proteção solar UVB (FPS-UVB) in vitro de uma série de azinas simétricas obtidas por meio de mecanoquímica, uma metodologia sintética favorável ao meio ambiente.

\section{Azinas acíclicas}

Compostos orgânicos contendo a estrutura geral $\mathrm{R}^{1} \mathrm{R}^{2} \mathrm{C}=\mathrm{N}-\mathrm{N}=\mathrm{CR}^{3} \mathrm{R}^{4}$ são denominados de azinas acíclicas, as quais são classificadas em cetazina $\left(\mathrm{R}^{1} \mathrm{R}^{2} \mathrm{C}=\mathrm{N}-\mathrm{N}=\mathrm{CR}^{3} \mathrm{R}^{4}\right)$ e aldazina $\left(\mathrm{R}^{1} \mathrm{HC}=\mathrm{N}-\mathrm{N}=\mathrm{CHR}^{2}\right)$. Tais compostos podem ser simétricos, quando os substituintes $R^{1}, R^{2}, R^{3}$ e $R^{4}$ são iguais, ou não simétricos, quando $\mathrm{R}^{1}, \mathrm{R}^{2}, \mathrm{R}^{3}$ e $\mathrm{R}^{4}$ são diferentes. ${ }^{6}$

As azinas acíclicas possuem três isômeros geométricos possíveis: $(E, E),(E, Z)$ e $(Z, Z)$. A possibilidade de arranjos moleculares distintos ocorre em função da configuração das ligações duplas $(\mathrm{C}=\mathrm{N})$ na cadeia de quatro átomos $(\mathrm{C}=\mathrm{N}-\mathrm{N}=\mathrm{C})$. Contudo, a maioria das azinas ocorre preferencialmente na configuração $(E, E)$, considerado o arranjo termodinamicamente mais estável. Na configuração $(E, E)$ os grandes grupos ligados às ligações $\mathrm{C}=\mathrm{N}$ estão na posição trans com relação à ligação $\mathrm{N}-\mathrm{N}$. ${ }^{7}$

As azinas substituídas com anéis aromáticos ou heterocíclicos são fotocrômicas e sofrem isomerização térmica e fotoquímica $E / Z$ sobre a dupla ligação $\mathrm{C}=\mathrm{N}$, conforme demonstrado na Figura 1. Esta propriedade torna as azinas acíclicas substâncias de interesse para o desenvolvimento de dosímetros de radiação ultravioleta, telas para proteção dos olhos, assim como, de dispositivos ópticos contra poderosas fontes emissoras de luz (explosões nucleares). ${ }^{8}$

Tratando-se de uma cadeia linear com ligações duplas conjugadas, esperava-se que azinas acíclicas diarílicas, adequadamente 


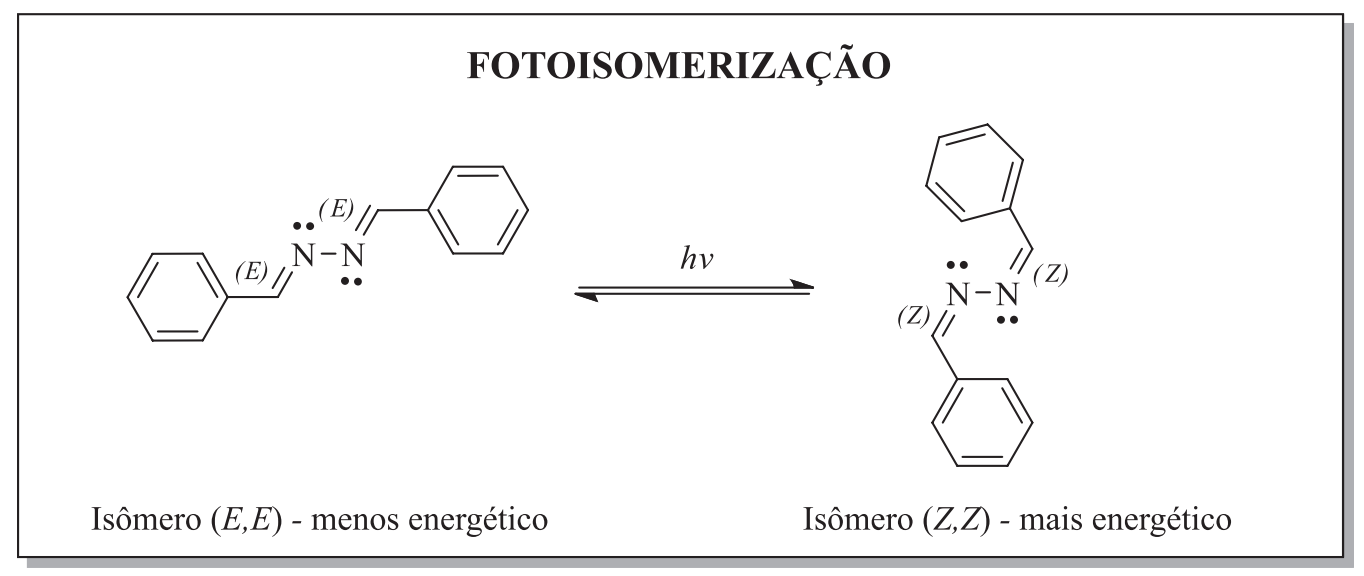

Figura 1. Isomerização fotoquímica de azinas acíclicas

substituídas, apresentassem um sistema estendido de deslocalização eletrônica. Contudo, estudos eletroquímicos ${ }^{9}$ e de Ressonância Magnética Nuclear $(\mathrm{RMN}){ }^{10}$ mostraram que este grupo funciona como um bloqueador de conjugação (Figura 2). Assim, as duas iminas $(\mathrm{C}=\mathrm{N})$ unidas pela ligação $\mathrm{N}-\mathrm{N}$, que constituem a função azina, podem ser consideradas grupos receptores de elétrons orientados em direções opostas, conforme descrito na Figura 2. Quando ligadas a dois anéis arílicos substituídos adequadamente, com grupos doadores ou receptores de elétrons, as azinas são capazes de gerar um momento de dipolo na estrutura química na molécula, o que as tornam candidatas apropriadas para o desenvolvimento de materiais ópticos não lineares. ${ }^{11}$

Além da relevância para obtenção de novos materais, as azinas são importantes intermediários na síntese de compostos heterocíclicos, como pirazóis, oxadiazóis, pirimidinas e purinas. ${ }^{12}$ Em adição, trabalhos têm relatado importantes atividades biológicas de compostos contendo este grupo funcional, com destaque para a atividade antitumoral ${ }^{13}$ e antimicrobiana. ${ }^{14}$

As azinas acíclicas simétricas são facilmente preparadas a partir da reação entre hidrazina e compostos carbonílicos (aldeídos ou cetonas), utilizando catalisadores como: $\mathrm{BiCl}_{3},{ }^{15}$ nano-argila de montmorilonita K10 funcionalizada com amina $\left(\mathrm{NH}_{2}-\mathrm{MMT}\right),{ }^{16}$ aduto de hidrazina e dióxido de carbono, ${ }^{17}$ meglumina, ${ }^{18}$ nanopartículas de $\mathrm{WCl}_{6}$ com montmorilonita $\mathrm{K}-10,{ }^{19} \mathrm{Al}_{2} \mathrm{O}_{3},{ }^{20}$ dietil fosfito, ${ }^{20}$ $\mathrm{FeCl}_{3} \cdot 6 \mathrm{H}_{2} \mathrm{O} .{ }^{21}$ Ademais, tem sido relatado o emprego de fontes alternativas de energia para obtenção de azinas acíclicas simétricas, como a de micro-ondas ${ }^{12}$ e de ultrassom. ${ }^{15}$ Outro método alternativo foi descrito no trabalho de Safari e Gandomi-Ravandi, no qual foi preparada uma série de azinas simétricas com altos rendimentos empregando energia mecânica gerada por moagem manual dos reagentes. ${ }^{22}$

\section{Mecanoquímica}

A síntese orgânica vem passando por mudanças significativas no sentido de desenvolver processos mais eficientes e sustentáveis. Assim, a denominada "química verde" tornou-se interesse dos pesquisadores de indústrias e instituições de ensino. A química verde fundamenta-se em 12 princípios gerais, sendo alguns deles: a diminuição do uso de solventes voláteis e tóxicos, a redução da quantidade de catalisadores e reagentes, a utilização de produtos químicos ambientalmente menos danosos, a redução da geração de resíduos químicos e a busca pela eficiência energética. ${ }^{23}$<smiles></smiles>

\section{BLOQUEIO DA CONJUGAÇÃO}

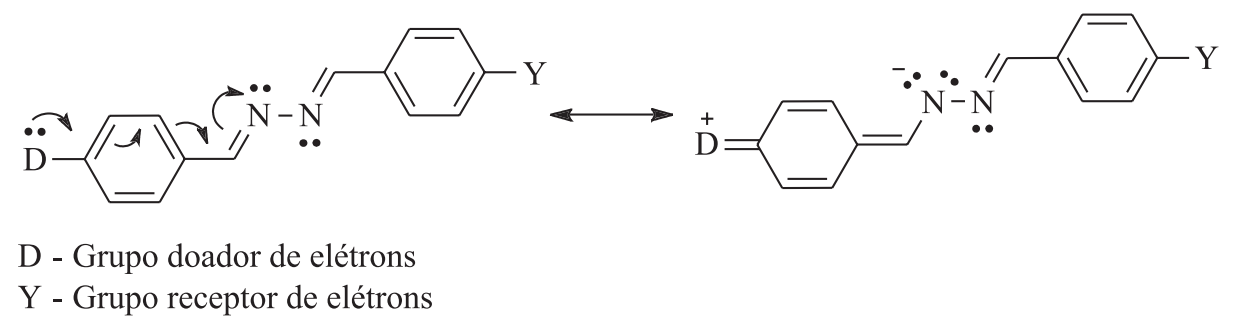

Figura 2. Conjugação das azinas acíclicas 
O interesse pelo desenvolvimento de metodologias de síntese que empreguem fontes alternativas de energia, como micro-ondas, força mecânica, luz visível e ultrassom vêm aumentando, e muitos resultados promissores já se encontram descritos na literatura. Nesse contexto, surge a mecanoquímica, que prima pela redução no uso de solvente e pela mínima geração de resíduos, bem como, pela eficiência energética na síntese de compostos orgânicos. ${ }^{24}$

Uma reação mecanoquímica é definida como uma reação química induzida por absorção direta de energia mecânica. Sendo ainda pouco conhecida, a mecanoquímica complementa, portanto, os métodos convencionais de ativação de algumas reações químicas, tais como o calor, a irradiação e a eletroquímica. ${ }^{25,26}$

É esperado que, a depender da fonte de energia empregada nas reações químicas, diferentes produtos possam ser gerados, inclusive, existem algumas reações que ocorrem na dependência de uma fonte de energia específica. ${ }^{25,27}$ Portanto, a mecanoquímica se apresenta não apenas como uma alternativa ecologicamente "amigável", mas também, trata-se de uma energia alternativa às convencionais, e que pode ser utilizada para promover reações químicas. Além de ser uma metodologia sintética sustentável, a mecanoquímica representa uma oportunidade para exploração de um novo campo de reações químicas, em que produtos não esperados para determinadas reações podem ser obtidos com a alternância da fonte energética empregada.

As primeiras reações mecanoquímicas foram realizadas usando gral e pistilo, o instrumento tradicional de moagem. ${ }^{27}$ No entanto, como tais reações se comportam a depender do operador, pois cada indivíduo pode imprimir diferentes quantidades de energia, e reações por longos períodos requerem resistência física, foram desenvolvidos dispositivos eletrônicos de moagem, ${ }^{25}$ como por exemplo, o moinho de bolas planetário.

Entendendo a mecanoquímica como alternativa eficaz e ambientalmente amigável, esta pode ser empregada para a obtenção de azinas acíclicas simétricas com potencialidades medicinais ou de gerar novos materiais.

\section{PARTE EXPERIMENTAL}

\section{Reagentes e equipamentos}

Todos os reagentes empregados foram obtidos de fonte comercial e utilizados sem purificação prévia. As substâncias obtidas tiveram suas temperaturas de fusão determinadas em triplicata empregando fusiômetro digital modelo MQAPF-302 da Microquímica Equipamentos LTDA. As Cromatografias em Camada Delgada Analítica (CCDA) foram realizadas em placas de alumínio de 2x4 cm recobertas com sílica gel 60 com indicador de fluorescência $U_{2} V_{254 m}$ e reveladas com luz ultravioleta (UV) ou iodo $\left(\mathrm{I}_{2}\right)$.

A elucidação estrutural das azinas foi realizada empregando-se as técnicas de Ressonância Magnética Nuclear de ${ }^{1} \mathrm{H} \mathrm{e}{ }^{13} \mathrm{C}(\mathrm{RMN}$ de ${ }^{1} \mathrm{H} \mathrm{e}{ }^{13} \mathrm{C}$ ), ${ }^{13} \mathrm{C}$-DEPT $135^{\circ}$, assim como, as técnicas bidimensionais de correlação homonuclear, ${ }^{1} \mathrm{H},{ }^{1} \mathrm{H}$-COSY, e heteronuclear, ${ }^{1} \mathrm{H},{ }^{13} \mathrm{C}$ (HSQC e HMBC). Os espectros de RMN foram registrados em um aparelho Bruker Ascend ${ }^{\mathrm{TM}} 400$, o qual opera a $400 \mathrm{MHz}$ para o núcleo de ${ }^{1} \mathrm{H}$ e a $100 \mathrm{MHz}$ para o núcleo de ${ }^{13} \mathrm{C}$. Os deslocamentos químicos ( $\delta$ ) foram obtidos em ppm utilizando o tetrametilsilano (TMS) como padrão interno. Todas as amostras foram solubilizadas em solvente deuterado $\left(\mathrm{CDCl}_{3}\right.$ ou DMSO-d $\left.\mathrm{d}_{6}\right)$.

Os espectros de absorção molecular na região do ultravioleta e visível foram obtidos usando um espectrofotômetro de absorção molecular (Nova Instruments 1600UV) e cubetas de quartzo.

Os espectros de absorção no infravermelho com transformada de Fourier (FTIR) foram obtidos utilizando o equipamento PerkinElmer ${ }^{\circledR}$ (Spectrum 400).

\section{Síntese e caracterização das azinas}

Em um almofariz foi misturado o aldeído adequado (1,0 mmol), o sulfato de hidrazina $(0,5 \mathrm{mmol})$ e a trietilamina $(0,5 \mathrm{mmol})$. Em seguida, a mistura foi triturada manualmente com auxílio de um pistilo em tempos que variaram de 5-10 minutos, e o progresso da reação foi acompanhado por CCDA. A pasta formada foi vertida em água gelada, e o sólido gerado foi filtrado e seco à temperatura ambiente. As azinas obtidas foram cristalizadas utilizando etanol e água deionizada.

\section{Benzaldazina (1)}

Sólido de coloração amarelo pálido esverdeado (Figura 1S); rendimento de 84\%; PF: $86-87{ }^{\circ} \mathrm{C}$; $\mathrm{RMN}$ de ${ }^{1} \mathrm{H}$ (400 $\left.\mathrm{MHz}, \mathrm{CDCl}_{3}\right)$ : $\delta \mathrm{ppm} 8,68(\mathrm{~s}, 2 \mathrm{H}, \mathrm{HC}=\mathrm{N}) ; 7,89-7,81(\mathrm{~m}, 4 \mathrm{H}, \mathrm{Ar}) ; 7,51-7,43(\mathrm{~m}$, $6 \mathrm{H}, \mathrm{Ar})$. RMN de ${ }^{13} \mathrm{C}\left(100 \mathrm{MHz}, \mathrm{CDCl}_{3}\right): \delta \mathrm{ppm} 162,2(\mathrm{C}=\mathrm{N}) ; 134,0$ $\left(\mathrm{N}=\mathrm{C}-\mathrm{C}_{\mathrm{Ar}}\right) ; 131,2\left(\mathrm{C}_{\mathrm{Ar}}\right) ; 128,8\left(\mathrm{C}_{\mathrm{Ar}}\right) ; 128,6\left(\mathrm{C}_{\mathrm{Ar}}\right) . \mathrm{IV}: \tilde{v} / \mathrm{cm}^{-1} 3047$ $\left.\left(\mathrm{C}-\mathrm{H} \mathrm{sp}^{2}\right) ; 2944(\mathrm{C}-\mathrm{H} \mathrm{sp})^{3}\right) ; 1623(\mathrm{C}=\mathrm{N}) ; 1578(\mathrm{C}=\mathrm{N} ; \mathrm{C}=\mathrm{N}-\mathrm{N}=\mathrm{C})$, Figuras 2S-8S.

\section{2-hidroxialdazina (2)}

Sólido de coloração amarelo pálido (Figura $1 \mathrm{~S}$ ); rendimento de 54\%; PF: $212-214{ }^{\circ} \mathrm{C}$; RMN de ${ }^{1} \mathrm{H}\left(400 \mathrm{MHz}, \mathrm{CDCl}_{3}\right): \delta \mathrm{ppm} 11,40$ (s, 2H, OH); 8,72 (s, 2H, HC=N); 7,44-7,33 (m, 4H, Ar); 7,04 (d, $J$ $=7,9 \mathrm{~Hz}, 2 \mathrm{H}, \mathrm{Ar}) ; 6,97$ (td, $J=7,6 ; 1,1 \mathrm{~Hz}, 2 \mathrm{H}, \mathrm{Ar}) . \mathrm{RMN} \mathrm{de}{ }^{13} \mathrm{C}$ $\left(100 \mathrm{MHz}, \mathrm{CDCl}_{3}\right): \delta[\mathrm{ppm}] 164,7(\mathrm{C}=\mathrm{N}) ; 159,8\left(\mathrm{OH}-\mathrm{C}_{\mathrm{Ar}}\right) ; 133,4$ $\left(\mathrm{C}_{\mathrm{Ar}}\right) ; 132,6\left(\mathrm{C}_{\mathrm{Ar}}\right) ; 119,7\left(\mathrm{C}_{\mathrm{Ar}}\right) ; 117,3\left(\mathrm{CH}-\mathrm{C}_{\mathrm{Ar}}\right) ; 117,1\left(\mathrm{C}_{\mathrm{Ar}}\right) . \mathrm{IV}$ : $\left.\tilde{v} / \mathrm{cm}^{-1} 3047(\mathrm{C}-\mathrm{H} \mathrm{sp})^{2}\right) ; 2950\left(\mathrm{C}-\mathrm{H} \mathrm{sp}^{3}\right) ; 1623(\mathrm{C}=\mathrm{N}) ; 1571(\mathrm{C}=\mathrm{N}$; $\mathrm{C}=\mathrm{N}-\mathrm{N}=\mathrm{C}$ ), Figuras $9 \mathrm{~S}-15 \mathrm{~S}$.

\section{4-hidroxialdazina (3)}

Sólido de coloração amarelo pálido (Figura $1 \mathrm{~S}$ ); rendimento de 69\%; PF: $230{ }^{\circ} \mathrm{C}$; RMN de ${ }^{1} \mathrm{H}\left(400 \mathrm{MHz}, \mathrm{DMSO}-\mathrm{d}_{6}\right): \delta \mathrm{ppm} 10,10$ (s, 2H, OH); 8,57 (s, 2H, HC=N); 7,70 (d, $J=8,7 \mathrm{~Hz}, 4 \mathrm{H}, \mathrm{Ar}) ; 6,87$ (d, $J=8,6 \mathrm{~Hz}, 4 \mathrm{H}, \mathrm{Ar}) . \mathrm{RMN}$ de ${ }^{13} \mathrm{C}\left(100 \mathrm{MHz}, \mathrm{DMSO}-\mathrm{d}_{6}\right): \delta \mathrm{ppm}$ $160,4(\mathrm{C}=\mathrm{N}) ; 160,3\left(\mathrm{OH}-\mathrm{C}_{\mathrm{Ar}}\right) ; 130,1\left(\mathrm{C}_{\mathrm{Ar}}\right) ; 125,1\left(\mathrm{CH}-\mathrm{C}_{\mathrm{Ar}}\right) ; 115,8$ $\left(\mathrm{C}_{\mathrm{Ar}}\right)$. IV: $\tilde{v} / \mathrm{cm}^{-1} 3022\left(\mathrm{C}-\mathrm{H} \mathrm{sp}^{2}\right) ; 2937\left(\mathrm{C}-\mathrm{H} \mathrm{sp}^{3}\right) ; 1623(\mathrm{C}=\mathrm{N}) ; 1597$ $(\mathrm{C}=\mathrm{N} ; \mathrm{C}=\mathrm{N}-\mathrm{N}=\mathrm{C})$, Figuras $16 \mathrm{~S}-22 \mathrm{~S}$.

\section{Vanilidazina (4)}

Sólido de coloração amarelo escuro (Figura 1S); rendimento de 70\%; PF: $172-176{ }^{\circ} \mathrm{C}$; RMN de ${ }^{1} \mathrm{H}\left(400 \mathrm{MHz}, \mathrm{DMSO}_{6}\right.$ ): $\delta$ ppm 8,74 $(\mathrm{s}, 2 \mathrm{H}, \mathrm{HC}=\mathrm{N}) ; 7,58(\mathrm{~d}, J=1,5 \mathrm{~Hz}, 2 \mathrm{H}, \mathrm{Ar}) ; 7,32(\mathrm{dd}, J=8,2 ; 1,7$ $\mathrm{Hz}, 2 \mathrm{H}, \mathrm{Ar}) ; 6,93$ (d, $J=8,1 \mathrm{~Hz}, 2 \mathrm{H}, \mathrm{Ar}) ; 3,85$ (s, 6H, $\mathrm{OCH}_{3}$ ). RMN de ${ }^{13} \mathrm{C}\left(100 \mathrm{MHz}, \mathrm{DMSO}-\mathrm{d}_{6}\right): \delta \mathrm{ppm} 160,9(\mathrm{C}=\mathrm{N}) ; 151,0\left(\mathrm{OH}-\mathrm{C}_{\mathrm{Ar}}\right)$; $148,1\left(\mathrm{CH}_{3} \mathrm{O}-\mathbf{C}_{\mathrm{Ar}}\right) ; 124,8\left(\mathrm{C}_{\mathrm{Ar}}\right) ; 124,2\left(\mathrm{CH}-\mathrm{C}_{\mathrm{Ar}}\right) ; 115,6\left(\mathrm{C}_{\mathrm{Ar}}\right) ; 110,4$ $\left(\mathrm{C}_{\mathrm{Ar}}\right) ; 55,6\left(\mathrm{OCH}_{3}\right)$. IV: $\left.\tilde{v} / \mathrm{cm}^{-1}: 3002\left(\mathrm{C}-\mathrm{H} \mathrm{sp}^{2}\right) ; 2931(\mathrm{C}-\mathrm{H} \mathrm{sp})^{3}\right) ; 1623$ $(\mathrm{C}=\mathrm{N}) ; 1597(\mathrm{C}=\mathrm{N} ; \mathrm{C}=\mathrm{N}-\mathrm{N}=\mathrm{C})$, Figuras 23S-29S.

\section{4-dimetilaminobenzoazina (5)}

Sólido de coloração amarelo escuro (Figura 1S); rendimento de 72\%; PF: $67-68{ }^{\circ} \mathrm{C}$; RMN de ${ }^{1} \mathrm{H}\left(400 \mathrm{MHz}, \mathrm{CDCl}_{3}\right)$ : $\delta$ ppm 8,58 (s, $2 \mathrm{H}, \mathrm{HC}=\mathrm{N}) ; 7,70(\mathrm{~d}, J=8,9 \mathrm{~Hz}, 4 \mathrm{H}, \mathrm{Ar}) ; 6,72(\mathrm{~d}, J=8,9 \mathrm{~Hz}, 4 \mathrm{H}$, $\mathrm{Ar}) ; 3,03$ (s, 12H, N $\left.\left(\mathrm{CH}_{3}\right)_{2}\right)$. RMN de ${ }^{13} \mathrm{C}\left(100 \mathrm{MHz}, \mathrm{CDCl}_{3}\right)$ : $\delta \mathrm{ppm}$ 160,8 $(\mathrm{C}=\mathrm{N}) ; 152,0\left(\mathrm{C}_{\mathrm{Ar}}-\mathrm{N}\left(\mathrm{CH}_{3}\right)_{2}\right) ; 129,8\left(\mathrm{C}_{\mathrm{Ar}}\right) ; 122,0\left(\mathrm{C}_{\mathrm{Ar}} \mathrm{C}=\mathrm{N}\right)$; 111,6 ( $\left.\mathrm{C}_{\mathrm{Ar}}\right) ; 40,2\left(\mathrm{~N}\left(\mathrm{CH}_{3}\right)_{2}\right)$. IV: $\tilde{v} / \mathrm{cm}^{-1}: 3040\left(\mathrm{C}-\mathrm{H} \mathrm{sp}^{2}\right) ; 2915(\mathrm{C}-\mathrm{H}$ $\left.\mathrm{sp}^{3}\right) ; 1660(\mathrm{C}=\mathrm{N}) ; 1364(\mathrm{C}-\mathrm{N})$, Figuras 30S-36S.

\section{Cinamaldazina (6)}

Sólido de coloração amarelo ouro (Figura $1 \mathrm{~S}$ ); rendimento de 70\%; PF: $161-163{ }^{\circ} \mathrm{C}$; RMN de ${ }^{1} \mathrm{H}\left(400 \mathrm{MHz}, \mathrm{DMSO}-\mathrm{d}_{6}\right)$ : $\delta \mathrm{ppm} 8,39$ $(\mathrm{d}, J=9,5 \mathrm{~Hz}, 2 \mathrm{H}, \mathrm{HC}=\mathrm{N}) ; 7,66(\mathrm{~d}, J=7,1 \mathrm{~Hz}, 4 \mathrm{H}, \mathrm{Ar}) ; 7,36-7,31$ (m, 8H, Ar); 7,15 (dd, $J=16,00 ; 9,50 \mathrm{~Hz}, 2 \mathrm{H}, \mathrm{HC}=\mathrm{CH}$ ). RMN de 
${ }^{13} \mathrm{C}\left(100 \mathrm{MHz}, \mathrm{DMSO}-\mathrm{d}_{6}\right): \delta$ ppm 163,2 (C=N); 143,4 (C=C); 135,6 $\left(\mathrm{CH}-\mathrm{C}_{\mathrm{Ar}}\right) ; 129,5\left(\mathrm{C}_{\mathrm{Ar}}\right) ; 129,0\left(\mathrm{C}_{\mathrm{Ar}}\right) ; 127,5\left(\mathrm{C}_{\mathrm{Ar}}\right) ; 125,3(\mathrm{C}=\mathrm{C})$. IV: $\left.\left.\tilde{v} / \mathrm{cm}^{-1}: 3040(\mathrm{C}-\mathrm{H} \mathrm{sp})^{2}\right) ; 2924(\mathrm{C}-\mathrm{H} \mathrm{sp})^{3}\right) ; 1630(\mathrm{C}=\mathrm{N}) ; 1584(\mathrm{C}=\mathrm{N}$; $\mathrm{C}=\mathrm{N}-\mathrm{N}=\mathrm{C}$ ), Figuras $37 \mathrm{~S}-43 \mathrm{~S}$.

\section{Determinação do Fator de Proteção Solar UVB in vitro}

\section{Preparação das formulações}

As formulações foram preparadas contendo o ativo (aldeídos, azinas ou filtros comerciais - benzofenona- 3 e metoxicinamato de octila) na concentração de 10,0\% (m/m). Inicialmente, $25 \mathrm{mg}$ de cada substância foi pesada, e posteriormente dispersa em $25 \mathrm{mg}$ de propilenoglicol sob agitação manual com auxílio de bastão de vidro. Por fim, foram adicionados $200 \mathrm{mg}$ de creme base Polawax ${ }^{\circledR}$ a 6,0\% (formulação base de protetor solar comercial) e homogeneizados sob agitação manual com bastão de vidro, Figura 1S. A formulação do "branco", utilizada para zerar o espectrofotômetro, foi preparada utilizando $25 \mathrm{mg}$ de propilenoglicol e $225 \mathrm{mg}$ de creme base. As formulações preparadas foram identificadas de acordo com a Tabela 1S.

\section{Determinação do fator de proteção solar UVB in vitro das formulações}

Foram preparados $50 \mathrm{~mL}$ de soluções etanólicas ( $96^{\circ} \mathrm{GL}$ ) com concentrações iniciais de $1,0 \times 10^{-3} \mathrm{~mol} \mathrm{~L}^{-1}$ de cada composto incorporado, utilizando as formulações preparadas especificadas na Tabela 1S. Para determinar o FPS-UVB in vitro das formulações foram preparadas soluções nas concentrações de $5,0 \times 10^{-5}$ e $2,5 \times 10^{-5} \mathrm{~mol} \mathrm{~L}^{-1}$, a partir da diluição da solução inicial $\left(1,0 \times 10^{-3} \mathrm{~mol} \mathrm{~L}^{-1}\right)$, sendo essas preparadas e analisadas em triplicata. Inicialmente, obteve-se o espectro de absorção UV-Vis característico de cada formulação, empregando-se a solução na concentração de $2,5 \times 10^{-5} \mathrm{~mol} \mathrm{~L}^{-1}$ e realizando leituras na faixa de comprimento de onda entre 200 e $450 \mathrm{~nm}$, com variação de $5 \mathrm{~nm}$. Em seguida, leituras espectrofotométricas foram realizadas para a solução de concentração $5,0 \times 10^{-5} \mathrm{~mol} \mathrm{~L}^{-1}$, nos comprimentos de onda de 290 a 320 nm. As absorbâncias obtidas foram aplicadas na equação determinada e descrita por Mansur e colaboradores, para as conversões aos respectivos valores de FPS-UVB, Equação $1 .^{28}$

$$
\mathrm{FPS}=\sum_{290}^{320} \times \mathrm{FC} \times \mathrm{EE}(\lambda) \times \mathrm{I}(\lambda) \times \operatorname{Abs}(\lambda)
$$

em que FC refere-se ao fator de correção (que assume valor 10); $\mathrm{EE}(\lambda)$ refere-se ao efeito eritematogênico da radiação em comprimento de onda específico; $\mathrm{I}(\lambda)$ refere-se à intensidade da radiação solar em cada comprimento de onda específico; e $\operatorname{Abs}(\lambda)$ refere-se à leitura espectrofotométrica da absorbância da amostra (solução etanólica da formulação) em cada comprimento de onda. Cabe salientar que os valores ponderais normalizados em função da ocorrência de eritemas na faixa UVB em cada comprimento de onda são valores resultantes do produto entre os parâmetros $\operatorname{EE}(\lambda)$ e $\mathrm{I}(\lambda)$, e seguem dispostos na Tabela $2 \mathrm{~S}^{29}$

\section{RESULTADOS E DISCUSSÃO}

\section{Síntese das azinas simétricas por moagem manual e caracterização estrutural}

Empregando energia mecânica, através de moagem manual, seis azinas simétricas foram sintetizadas na ausência de solventes a partir de aldeídos aromáticos e sulfato de hidrazina, empregando trietilamina como catalisador. Os rendimentos das reações foram satisfatórios (54-84\%), e a água e o etanol, solventes verdes, foram usados na precipitação e purificação dos produtos, Tabela 1 .

A obtenção das seis azinas simétricas, com bons rendimentos e empregando os princípios da macanoquímica e Química Verde, permite que essa síntese seja enquadrada no chamado Princípio de Price, em referência a Barry Price, inventor da ranitidina, o qual preconiza o uso de metodologias de baixo custo e alta reprodutibilidade. ${ }^{30}$

As azinas sintetizadas tiveram suas estruturas moleculares caracterizadas através da espectroscopia de RMN de ${ }^{1} \mathrm{H} \mathrm{e}{ }^{13} \mathrm{C}(400$ e $100 \mathrm{MHz}, \mathrm{CDCl}_{3}$ ), incluindo as técnicas bidimensionais ${ }^{1} \mathrm{H}$, ${ }^{1} \mathrm{H}-\mathrm{COSY},{ }^{1} \mathrm{H},{ }^{13} \mathrm{C}$ (HSQC e HMBC) e ${ }^{13} \mathrm{C}$-DEPT $135^{\circ}$, em que todos os carbonos e hidrogênios tiveram os deslocamentos químicos atribuídos. Além da caracterização por RMN, foram obtidos os espectros de absorção na região do IV.

O espectro de $\mathrm{RMN}$ de ${ }^{13} \mathrm{C}\left(100 \mathrm{MHz}, \mathrm{CDCl}_{3}\right)$ da benzaldazina (1) (Figura 3S) apresentou 5 linhas espectrais. Através da análise comparativa dos espectros de RMN de ${ }^{13} \mathrm{C}$ e ${ }^{13} \mathrm{C}$-DEPT $135^{\circ}$ (100 $\mathrm{MHz}, \mathrm{CDCl}_{3}$ ) (Figura 4S) foi possível determinar a presença de quatro sinais de carbono-monoidrogenado $(\mathrm{CH})$, todos eles localizados na região de carbono com hibridização $\mathrm{sp}^{2} \mathrm{e}$ na região de carbono de anel aromático, sendo o sinal em $\delta_{\mathrm{C}} 162,2$ referente aos carbonos $\mathrm{C}=\mathrm{N}$. Também foi identificado um sinal de carbono não hidrogenado na região de hibridização $\mathrm{sp}^{2}$ em $\delta_{\mathrm{C}} 134,0$.

Tabela 1. Azinas acíclicas simétricas sintetizadas com rendimentos e ponto de fusão obtidos experimentalmente

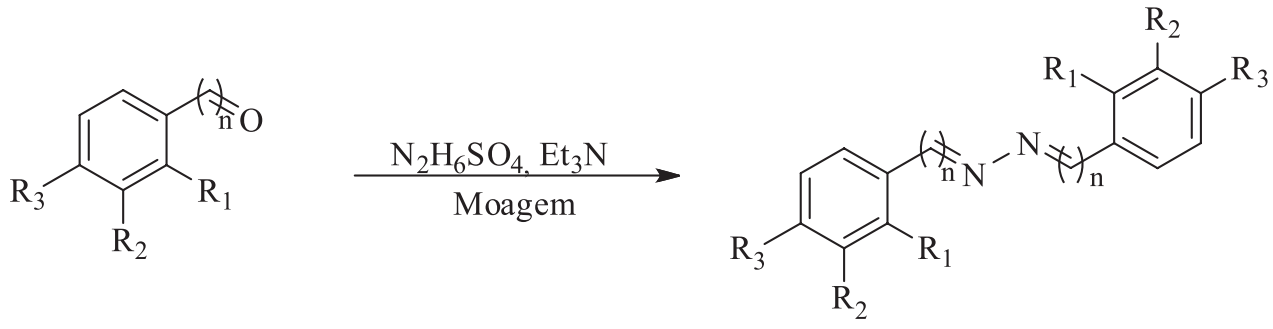

\begin{tabular}{|c|c|c|c|c|c|c|}
\hline AZINAS & $\mathrm{R}_{1}$ & $\mathrm{R}_{2}$ & $\mathrm{R}_{3}$ & $\mathrm{n}$ & Rend. $(\%)$ & $\mathrm{PF}\left({ }^{\circ} \mathrm{C}\right)$ \\
\hline Benzaldazina (1) & $\mathrm{H}$ & $\mathrm{H}$ & $\mathrm{H}$ & 1 & 84 & $86-87$ \\
\hline 2-Hidroxialdazina (2) & $\mathrm{OH}$ & $\mathrm{H}$ & $\mathrm{H}$ & 1 & 54 & $212-214$ \\
\hline 4-Hidroxialdazina (3) & $\mathrm{H}$ & $\mathrm{H}$ & $\mathrm{OH}$ & 1 & 69 & 230 \\
\hline Vanilidazina (4) & $\mathrm{H}$ & $\mathrm{OCH}_{3}$ & $\mathrm{OH}$ & 1 & 70 & $172-176$ \\
\hline 4-Dimetilaminobenzoazina (5) & $\mathrm{H}$ & $\mathrm{H}$ & $\mathrm{N}\left(\mathrm{CH}_{3}\right)_{2}$ & 1 & 72 & $67-68$ \\
\hline Cinamaldazina (6) & $\mathrm{H}$ & $\mathrm{H}$ & $\mathrm{H}$ & 3 & 70 & $161-163$ \\
\hline
\end{tabular}


Analisando o espectro de RMN de ${ }^{1} \mathrm{H}\left(400 \mathrm{MHz}, \mathrm{CDCl}_{3}\right)$ da benzaldazina (1) (Figura 2S) foram observados sinais na região de hidrogênio aromático, os multipletos em $\delta_{\mathrm{H}} 7,89-7,81$ e $\delta_{\mathrm{H}} 7,51-7,43$, referentes a dez hidrogênios; e um simpleto em uma região mais desblindada do espectro, em $\delta_{\mathrm{H}} 8,68$, com integral para dois hidrogênios, o qual é referente aos hidrogênios do grupo funcional aldazina $(\mathrm{HRC}=\mathrm{N}-\mathrm{N}=\mathrm{CRH})$. No espectro bidimensional de correlação heteronuclear direta, ${ }^{1} \mathrm{H},{ }^{13} \mathrm{C}$-HSQC (400 MHz, $\mathrm{CDCl}_{3}$ ) (Figura S6), foi observada a correlação entre o sinal em $\delta_{\mathrm{H}} 8,68$, referente aos hidrogênios azínicos, com o sinal de carbono em $\delta_{\mathrm{C}} 162,2$. Por fim, a ausência de sinal de carbonila $\left(\delta_{\mathrm{C}} 185,0-220,0\right)$ e hidrogênio $\left(\delta_{\mathrm{H}} 9,00-10,00\right)$ aldeídico ratifica o sucesso da reação. ${ }^{30}$

Diferentemente do espectro da benzaldazina (1), o espectro de $\mathrm{RMN} \mathrm{de}{ }^{13} \mathrm{C}\left(100 \mathrm{MHz}, \mathrm{CDCl}_{3}\right)$ da 2-hidroxialdazina (2) (Figura 10S) apresentou dois sinais para carbono não hidrogenado em $\delta_{\mathrm{C}} 117,3 \mathrm{e}$ 159,8 , estes são referentes aos carbonos, dos anéis aromáticos ligados aos grupos azina e hidroxila, respectivamente.

No espectro de RMN de ${ }^{1} \mathrm{H}\left(400 \mathrm{MHz}, \mathrm{CDCl}_{3}\right.$ ) da 2-hidroxialdazina (2) (Figura 9S) foi possível observar dois simpletos em região desblindada $\left(\delta_{\mathrm{H}} 11,40\right.$ e 8,72$)$, cada um referente a dois hidrogênios; além dos sinais em $\delta_{\mathrm{H}} 7,44-7,33$, em $\delta_{\mathrm{H}} 7,04$ e em $\delta_{\mathrm{H}} 6,97$, relativos aos hidrogênios aromáticos. Conforme o espectro ${ }^{1} \mathrm{H},{ }^{13} \mathrm{C}-\mathrm{HSQC}(400 \mathrm{MHz}$, $\mathrm{CDCl}_{3}$ ) (Figura 13S), os hidrogênios em $\delta_{\mathrm{H}} 11,40$ não são ligados diretamente a carbono, enquanto que os hidrogênios em $\delta_{\mathrm{H}} 8,72$ acoplam diretamente com o sinal de carbono azínico $\left(\delta_{\mathrm{C}} 164,7\right)$. Além disso, no espectro de correlação heteronuclear ${ }^{1} \mathrm{H},{ }^{13} \mathrm{C}-\mathrm{HMBC}\left(400 \mathrm{MHz}, \mathrm{CDCl}_{3}\right)$ (Figura 14S), o qual possibilita correlacionar os átomos de hidrogênio e carbono a duas $\left({ }^{2} J_{\mathrm{C}-\mathrm{H}}\right)$ e três $\left({ }^{3} J_{\mathrm{C}-\mathrm{H}}\right)$ ligações, foi possível observar a correlação entre os sinais do hidrogênio em $\delta_{\mathrm{H}} 8,72$ com os carbonos em $\delta_{\mathrm{C}} 117,3,132,6$ e 159,8 , Figura 3.

$\mathrm{O}$ espectro de RMN de ${ }^{13} \mathrm{C}\left(100 \mathrm{MHz}, \mathrm{DMSO}-\mathrm{d}_{6}\right)$ da 4-hidroxialdazina (3) (Figura 17S) apresentou dois sinais de carbono não hidrogenado em $\delta_{\mathrm{C}} 160,3$ e 125,1 , e três sinais de carbono monoidrogenado, um deles em $\delta_{\mathrm{C}} 160,4$, referente aos carbonos $\mathrm{C}=\mathrm{N}$. No espectro de RMN de ${ }^{1} \mathrm{H}\left(400 \mathrm{MHz}, \mathrm{DMSO}-\mathrm{d}_{6}\right)$ da 4-hidroxialdazina (3) (Figura 16S) observou-se dois simpletos em região desblindada $\left(\delta_{\mathrm{H}} 10,10\right.$ e 8,57$)$, cada um com integral para dois hidrogênios, além de dois dubletos em $\delta_{\mathrm{H}} 7,70$ e $6,87, \operatorname{com} J=8,6 \mathrm{~Hz}$, cada um referente a quatro hidrogênios, o que comprova a relação para entre os substituintes dos anéis aromáticos. O espectro ${ }^{1} \mathrm{H},{ }^{13} \mathrm{C}-\mathrm{HSQC}(400 \mathrm{MHz}$, DMSO-d ${ }_{6}$ ) (Figura 20S) revela que os hidrogênios em $\delta_{\mathrm{H}} 10,10$ não se encontram ligados a carbonos, correspondendo, portanto, aos hidrogênios das hidroxilas $p$-substituintes. Ademais, os hidrogênios em $\delta_{\mathrm{H}} 8,57$ acoplam com o sinal de carbono azínico $\left(\delta_{\mathrm{C}} 160,4\right)$. No espectro ${ }^{1} \mathrm{H},{ }^{13} \mathrm{C}-\mathrm{HMBC}\left(400 \mathrm{MHz}, \mathrm{CDCl}_{3}\right)$ (Figura 21S) foi observada a relação entre os hidrogênios em $\delta_{\mathrm{H}} 8,57$ com os sinais de carbono em $\delta_{\mathrm{c}} 130,1$ e 125,1 , Figura 3.

No espectro de RMN de ${ }^{13} \mathrm{C}\left(100 \mathrm{MHz}, \mathrm{DMSO}-\mathrm{d}_{6}\right)$ da vanilidazina (4) (Figura 24S) foi possível identificar três sinais de carbono não hidrogenado em $\delta_{\mathrm{C}} 151,0,148,1$ e 124,2, quatro de carbono monoidrogenado e um de carbono com hibridização $\mathrm{sp}^{3}$ em $\delta_{\mathrm{C}} 55,6$.

$\mathrm{O}$ espectro de $\mathrm{RMN}$ de ${ }^{1} \mathrm{H}\left(400 \mathrm{MHz}, \mathrm{DMSO}-\mathrm{d}_{6}\right)$ da vanilidazina (4) (Figura 23S), por sua vez, revelou cinco sinais, sendo eles: um simpleto em $\delta_{\mathrm{H}} 8,74$, com integral para dois hidrogênios, dois dubletos em $\delta_{\mathrm{H}} 7,58$ e 6,93 e um duplo dubleto em $\delta_{\mathrm{H}} 7,32$, os três últimos com integral para dois hidrogênios cada. Além desses, foi observado um simpleto em $\delta_{\mathrm{H}} 3,85$ com integral para seis hidrogênios. No espectro ${ }^{1} \mathrm{H},{ }^{13} \mathrm{C}-\mathrm{HSQC}\left(400 \mathrm{MHz}\right.$, DMSO-d ${ }_{6}$ ) (Figura $27 \mathrm{~S}$ ) foi observado que os hidrogênios em $\delta_{\mathrm{H}} 8,74$ e 3,85 correlacionam com carbonos em $\delta_{\mathrm{C}} 160,9$ e 55,6 , respectivamente, enquanto que no espectro ${ }^{1} \mathrm{H},{ }^{13} \mathrm{C}-\mathrm{HMBC}\left(400 \mathrm{MHz}, \mathrm{DMSO}-\mathrm{d}_{6}\right.$ ) (Figura 28S) foi detectada a correlação entre os sinais de hidrogênio em $\delta_{\mathrm{H}} 8,74 \mathrm{com}$ os carbonos em $\delta_{\mathrm{C}} 124,8,124,2$ e 110,4 , Figura 3 .
O espectro de RMN de ${ }^{13} \mathrm{C}\left(100 \mathrm{MHz}, \mathrm{CDCl}_{3}\right)$ da 4-dimetilaminobenzoazina (5) (Figura 31S) apresentou dois sinais de carbono não hidrogenado, em $\delta_{\mathrm{C}} 152,0$ e 122,0, referentes aos carbonos aromáticos substituídos. Também foi possível identificar três sinais de carbono monoidrogenado com hibridização $\mathrm{sp}^{2}$, um deles $\left(\delta_{\mathrm{C}} 160,8\right)$ sendo referente aos carbonos $\mathrm{C}=\mathrm{N}$. Ademais, em $\delta_{\mathrm{C}} 40,2$, foi observado o sinal das metilas do grupo $\mathrm{N}\left(\mathrm{CH}_{3}\right)_{2}$.

$\mathrm{O}$ espectro de RMN de ${ }^{1} \mathrm{H}\left(400 \mathrm{MHz}, \mathrm{CDCl}_{3}\right)$ da 4-dimetilaminobenzoazina (5) (Figura 30S) exibiu dois simpletos em $\delta_{\mathrm{H}} 8,58(2 \mathrm{H})$ e 3,03 (12H); além de dois dubletos em $\delta_{\mathrm{H}} 7,70$ e 6,72, com integral para 4 hidrogênios cada. O espectro ${ }^{1} \mathrm{H},{ }^{13} \mathrm{C}$-HSQC ( $400 \mathrm{MHz}, \mathrm{CDCl}_{3}$ ) (Figura 34S), por sua vez, mostrou a ligação direta entre os hidrogênios em $\delta_{\mathrm{H}} 8,58$ com o sinal de carbono em $\delta_{\mathrm{C}} 160,8$, confirmando dessa maneira os sinais dos hidrogênios e carbonos do grupo azina. No espectro de correlação heteronuclear a $\mathrm{a}^{2,3} \mathrm{~J}_{\mathrm{C}-\mathrm{H}},{ }^{1} \mathrm{H},{ }^{13} \mathrm{C}-\mathrm{HMBC}(400$ $\mathrm{MHz}, \mathrm{CDCl}_{3}$ ) (Figura 35S), foi possível verificar a correlação entre os hidrogênios em $\delta_{\mathrm{H}} 3,03$ e o sinal de carbono em $\delta_{\mathrm{C}} 152,0$, e entre os hidrogênios em $\delta_{\mathrm{H}} 8,58$ com os sinais em $\delta_{\mathrm{C}} 122,1$ e 129,8, Figura 3 .

A cinamaldazina (6) apresentou sete sinais no espectro de RMN de ${ }^{13} \mathrm{C}\left(100 \mathrm{MHz}\right.$, DMSO-d ${ }_{6}$ ) (Figura 38S). Foi possível determinar a presença de um sinal de carbono não hidrogenado, em $\delta_{\mathrm{C}} 135,6$, e seis de carbono monoidrogenado, um deles em $\delta_{\mathrm{C}} 163,2$, o qual é referente aos carbonos $\mathrm{C}=\mathrm{N}$. Por sua vez, o espectro de $\mathrm{RMN}$ de ${ }^{1} \mathrm{H}$ (400 MHz, DMSO-d $_{6}$ da cinamaldazina (6) (Figura 37S) mostrou quatro sinais, dois dubletos, em $\delta_{\mathrm{H}} 8,39$ e 7,66, um mutltipleto, em $\delta_{\mathrm{H}} 7,36-7,31$, e um duplo dubleto em $\delta_{\mathrm{H}} 7,15$, com integração respectivamente de 2:4:8:2. O espectro bidimensional de correlação homonuclear, ${ }^{1} \mathrm{H},{ }^{1} \mathrm{H}-\mathrm{COSY}\left(400 \mathrm{MHz}, \mathrm{DMSO}-\mathrm{d}_{6}\right.$ ) (Figura 40S), apresenta a relação entre os hidrogênios em $\delta_{\mathrm{H}} 8,39$ e 7,15. Já o espectro ${ }^{1} \mathrm{H},{ }^{13} \mathrm{C}-\mathrm{HSQC}\left(400 \mathrm{MHz}, \mathrm{DMSO}-\mathrm{d}_{6}\right.$ ) (Figura 41S) revela a ligação direta entre os hidrogênios em $\delta_{\mathrm{H}} 8,39$ com o sinal de carbono em $\delta_{\mathrm{C}} 163,2$, e dos hidrogênios em $\delta_{\mathrm{H}} 7,15$ com o sinal em $\delta_{\mathrm{C}} 125,3$. No espectro ${ }^{1} \mathrm{H},{ }^{13} \mathrm{C}-\mathrm{HMBC}\left(400 \mathrm{MHz}, \mathrm{DMSO}-\mathrm{d}_{6}\right)$ (Figura 42S) é revelada a correlação entre os hidrogênios em $\delta_{\mathrm{H}} 7,15$ com os carbonos em $\delta_{\mathrm{C}} 135,6$ e 143,4 , Figura 3 .

As bandas de absorção observadas no espectro de IV das azinas corroboram com os dados dos experimentos de RMN. A banda referente à ligação $\mathrm{C}=\mathrm{N}$ foi observada em $1623 \mathrm{~cm}^{-1}$ nos espectros da benzaldazina (1), 2-hidroxialdazina (2), 4-hidroxialdazina (3) e vanilidazina (4) (Figuras 8S, 15S, 22S, 29S). O mesmo grupo apresentou bandas de absorção em $1660 \mathrm{~cm}^{-1}$ e $1630 \mathrm{~cm}^{-1}$, nos espectros da 4-dimetilaminobenzoazina (5) (Figura 36S) e cinamaldazina (6) (Figura 43S), respectivamente. ${ }^{31,32}$

\section{Preparação das formulações e determinação do FPS-UVB}

Com a confirmação das estruturas moleculares dos compostos sintéticos por espectroscopia de RMN e IV, as formulações fotoprotetoras $10,0 \%(\mathrm{~m} / \mathrm{m})$ foram adequadamente preparadas conforme relatado na metodologia. As cores adquiridas pelas formulações após a incorporação dos compostos testados foram semelhantes à coloração original dos compostos orgânicos, conforme Figura 1S.

Para fins de organização, as formulações fotoprotetoras contendo os aldeídos precursores, as azinas sintetizadas e os filtros comerciais foram nomeadas conforme: BDZ (benzaldeído); AZN-BDZ (benzaldazina); 2-OH (2-hidroxibenzaldeído); AZN-2OH (2-hidroxibenzaldazina); 4-OH (4-hidroxibenzaldeído); AZN-4OH (4-hidroxibenzaldazina); VAN (vanilina); AZN-VAN (vanilidazina); CIN (cinamaldeído); AZN-CIN (cinamaldazina); 4DAB (4-dimetilaminobenzaldeído); AZN-4DAB (4-dimetilaminobenzoazina); BZF (benzofenona) e OMC (metoxicinamato de octila).

Com as formulações devidamente preparadas foi possível obter os espectros de absorção molecular na faixa de comprimento de onda 


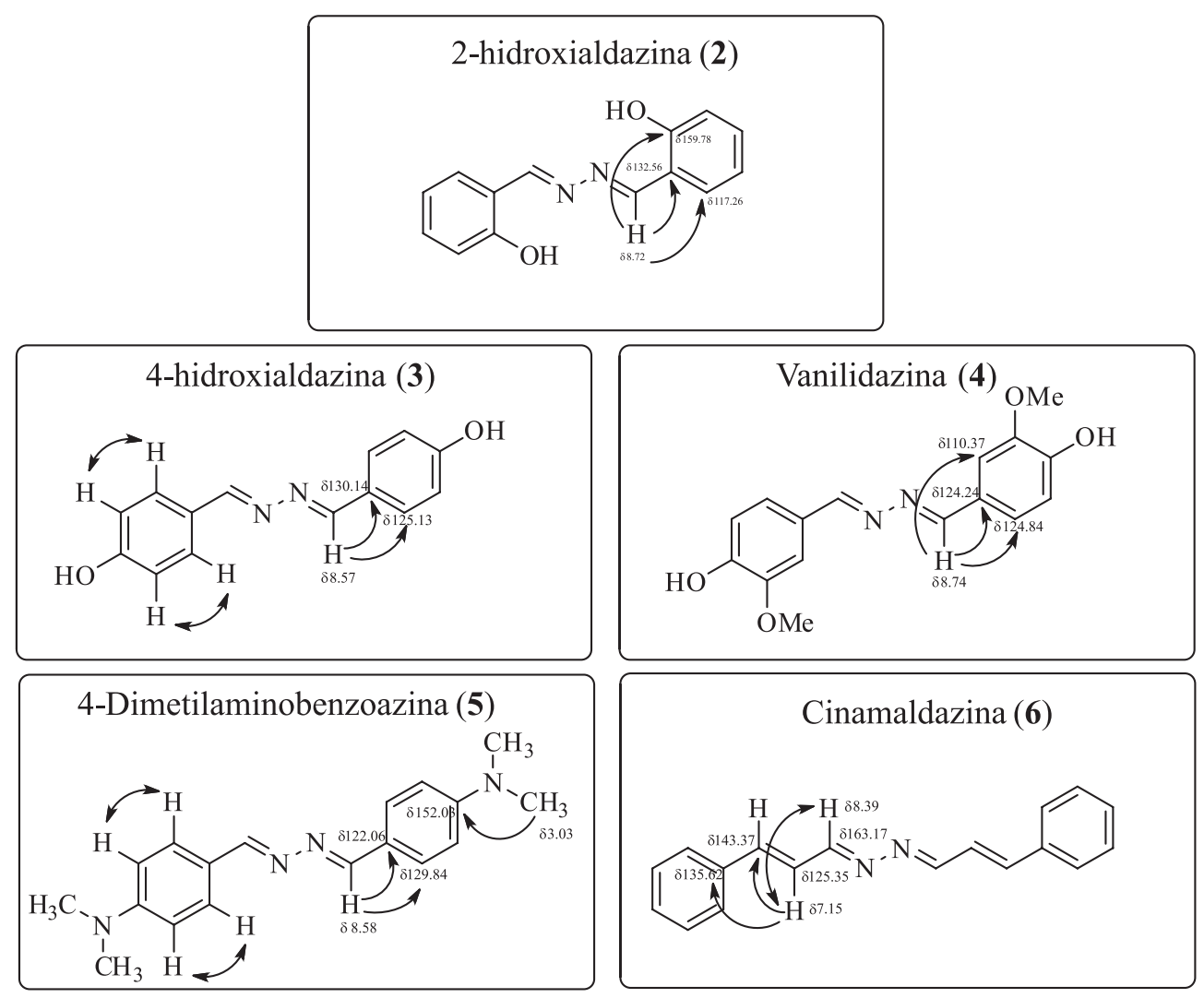

Figura 3. Representação das principais correlações que confirmaram a estrutura molecular das azinas sintetizadas (2-6). As setas simples representam o acoplamento ${ }^{1} \mathrm{H}^{13} \mathrm{C}$ a ${ }^{2,3} \mathrm{~J}_{\mathrm{CH}}$ e as setas duplas representam o acoplamento entre os hidrogênios vicinais

situada entre 200 e $450 \mathrm{~nm}$. Para fins de comparação, também foi obtido o espectro de absorção na região do UV/Vis das formulações contendo os aldeídos precursores das azinas e os filtros químicos comerciais benzofenona-3 (BZF) e metoxicinamato de octila (OMC). $\mathrm{O}$ resultado desse ensaio pode ser visto na Figura 4.

As formulações BZF e OMC apresentam bandas de máxima absorção $\left(\lambda_{\max }\right)$ nos comprimentos de onda de 280 e $325 \mathrm{~nm}$, para a primeira, e 230 e $310 \mathrm{~nm}$ para a segunda, corroborando com a literatura. ${ }^{33,34} \mathrm{~A} A Z N-B Z D$ e a AZN-4OH absorveram principalmente na região UVB, com $\lambda_{\max }$ em 300 e $280 \mathrm{~nm}$, respectivamente, enquanto que a AZN-VAN, a AZN-CIN e a AZN-4DAB absorveram principalmente na região UVA, com $\lambda_{\max }$ em 335, 345 e $390 \mathrm{~nm}$, respectivamente. A AZN-2OH, apresentou capacidade de absorção significativa tanto na região UVB ( $\left.\lambda_{\max } 295 \mathrm{~nm}\right)$ quanto na UVA $\left(\lambda_{\max } 355 \mathrm{~nm}\right)$.

O FPS-UVB das formulações foi determinado pelo método in vitro desenvolvido por Mansur e colaboradores. ${ }^{28}$ Santos e colaboradores compararam o FPS de formulações contendo OMC empregando o método desenvolvido por Mansur e o método in vivo preconizado pelo Food and Drug Administration (FDA), e concluíram que há uma boa relação entre os resultados obtidos pelos dois métodos. ${ }^{35}$ Ademais, considerando que o método in vivo de avaliação do FPS-UVB é realizado em seres humanos, iniciar os estudos usando previamente um método in vitro para testar a capacidade fotoprotetora de novos filtros químicos é um protocolo que deve ser estimulado.

Com a finalidade de se determinar os valores de FPS-UVB das formulações preparadas, foram realizadas as leituras em triplicata, no espectrofotômetro UV/Vis na região de 290-320 nm, e os FPS-UVB calculados de acordo com a Equação 1. Os resultados obtidos estão expressos na Figura 5, com valores de FPS \pm Desvio Padrão (DP).

Ao analisar os valores de FPS-UVB obtidos na concentração de $5,0 \times 10^{-5} \mathrm{~mol} \mathrm{~L}^{-1}$, nota-se que todas as formulações contendo as azinas sintetizadas apresentaram FPS-UVB superiores ao filtro de referência
$\mathrm{BZF}(3,83 \pm 0,08)$. Já comparando os respectivos FPS-UVB das azinas sintetizadas com o do $\mathrm{OMC}(9,43 \pm 0,13)$, um filtro solar orgânico com maior capacidade de absorção de radiação UV, a AZN-VAN $(6,66 \pm 0,40)$ e a AZN-4DAB $(7,08 \pm 0,01)$ apresentaram atividade fotoprotetora inferior. E ao analisar o espectro de absorção molecular do AZN-4DAB percebe-se que esta azina absorve com mais eficiência na região UVA, enquanto que o OMC apresenta absorção tanto na região do UVA quanto do UVB, Figura 4.

O OMC é um filtro solar orgânico que sofre fotoisomerização, no qual o isômero $E$ absorve radiação UV e é convertido ao isômero $Z$ (mais energético). Durante o retorno à configuração mais estável há liberação de radiação infravermelha e visível em um processo contínuo. ${ }^{34}$ Considerando que as azinas funcionam como bloqueador de conjugação e sofrem fotoisomerização, possivelmente a absorção de radiação ultravioleta pelas azinas simétricas (1-6) sintetizadas no presente trabalho ocorre pela isomerização dos compostos de sua forma menos energética $E / E$ para as formas mais energéticas $E / Z$ e $Z / Z$.

Para avaliar a influência do grupo funcional azina no fator de proteção solar UVB foram determinados os valores de FPS-UVB de formulações na concentração de $10,0 \%(\mathrm{~m} / \mathrm{m})$ dos aldeídos empregados como reagente de partida na síntese das azinas. Como pode ser observado na Figura 6, as formulações contendo os aldeídos não apresentaram FPS-UVB significativos, com exceção da CIN,

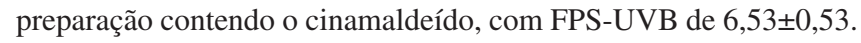

Comparando-se os espectros de varredura UV-Vis das azinas e dos aldeídos precursores (Figura 4) foi observado que a introdução do grupo azina na molécula promoveu um deslocamento batocrômico e um efeito hipercrômico no perfil de absorção molecular dos aldeídos utilizados, Figura 4. Esses deslocamentos favoreceram o aumento do FPS-UVB em todas as formulações contendo as azinas simétricas (1-6), Figura 6. Dessa forma, foi constatado que as azinas simétricas sintetizadas por mecanoquímica são moléculas com maior capacidade 

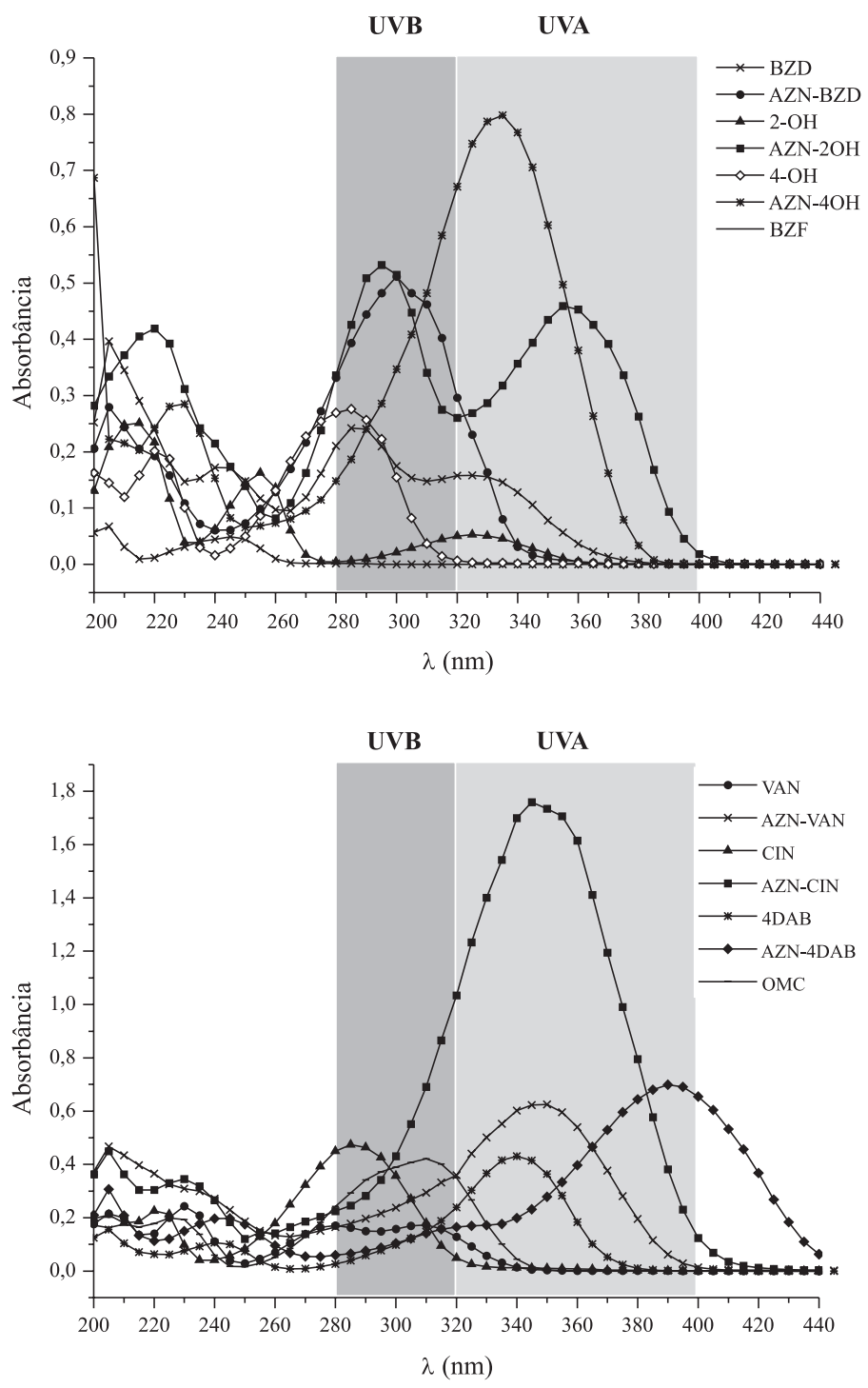

Figura 4. Espectros de absorção molecular UV/Vis das formulações contendo os aldeídos precursores, as azinas sintetizadas e os filtros comerciais na concentração de $2,5 \times 10^{-5} \mathrm{~mol} \mathrm{~L}^{-1}$ empregando-se etanol $96^{\circ} \mathrm{GL}$

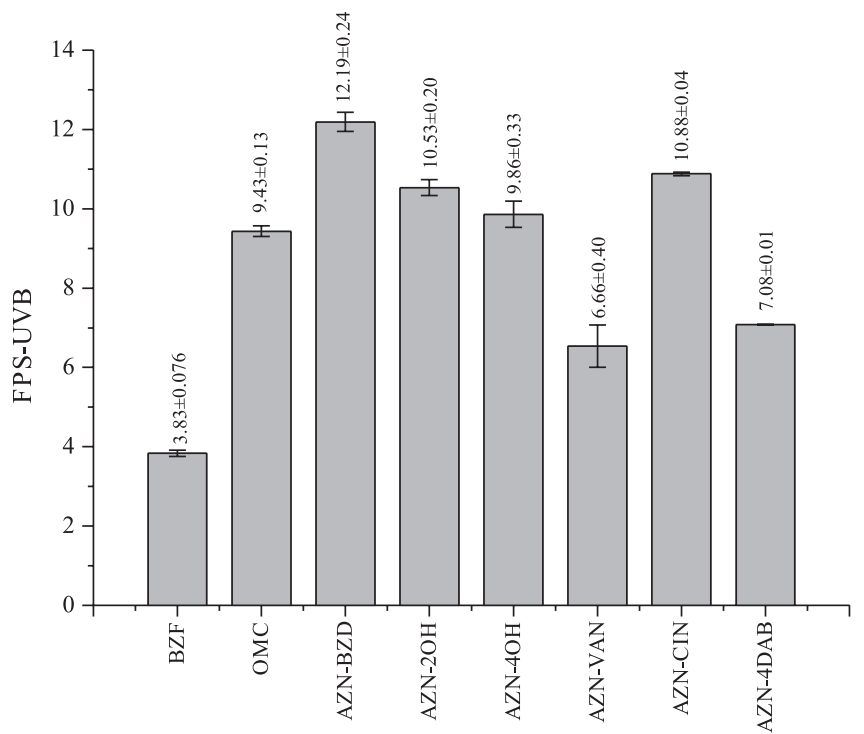

Figura 5. FPS-UVB \pm DP obtido a partir de soluções etanólicas das formulações contendo as azinas sintéticas e os filtros químicos orgânicos BZF e OMC na concentração de 5,0 $\times 10^{-5} \mathrm{~mol} \mathrm{~L}^{-1}$

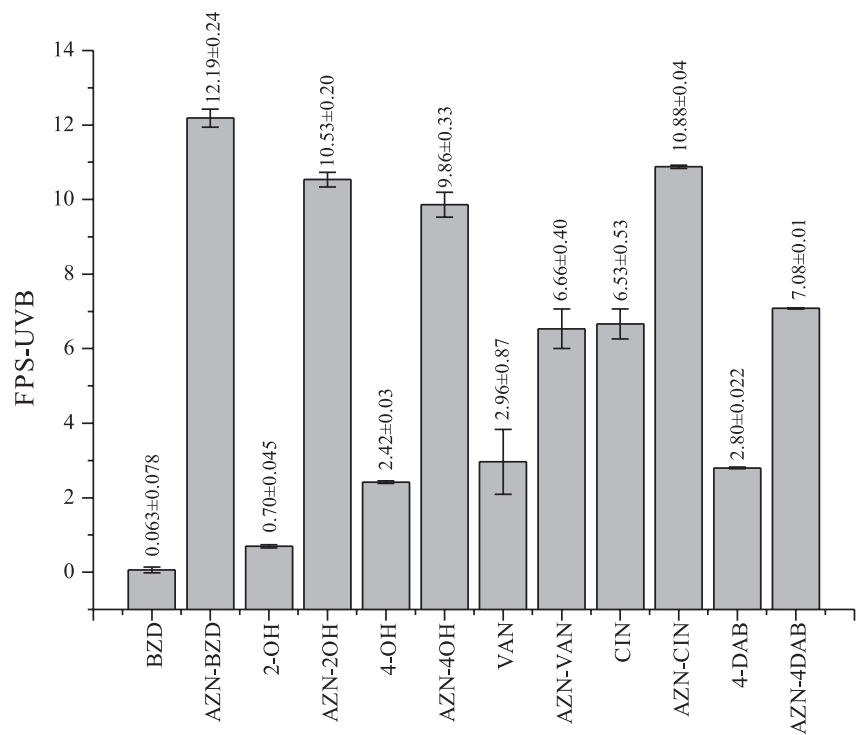

Figura 6. FPS-UVB \pm DP obtido a partir de soluções etanólicas das formulações contendo as azinas sintéticas e os aldeídos precursores na concentração de $5,0 \times 10^{-5} \mathrm{~mol} \mathrm{~L}^{-1}$ 
fotoprotetora que seus aldeídos precursores, e apresentam FPS-UVB comparável ou superior a dois dos principais filtros químicos comerciais, a BZF e o OMC.

\section{CONCLUSÃO}

Neste trabalho foram sintetizadas seis azinas acíclicas simétricas, empregando-se a moagem manual como fonte de energia, com rendimentos reacionais satisfatórios, entre 54 e $84 \%$. Também foram preparadas formulações fotoprotetoras contendo as azinas sintetizadas, sendo que quatro apresentaram FPS-UVB maior que a formulação contendo o metoxicinamato de octila (OMC), o filtro químico comercial adotado como referencial comparativo, fato que demonstrou a alta capacidade fotoprotetora desta série de azinas. As formulações AZN-VAN e AZN-4DAB contendo, respectivamente, vanilidazina e 4-dimetilaminobenzoazina, foram as que apresentaram menor FPS-UVB, porém, observa-se que essas duas formulações apresentam maior capacidade de absorver radiação UVA. Sendo assim, os valores de FPS-UVB obtidos para as formulações preparadas, em associação com as vantagens de ter sido adotado um método sintético ambientalmente favorável e de baixo custo para a obtenção dos produtos almejados, tornam as azinas candidatas promissoras a novos filtros solares orgânicos.

\section{MATERIAL SUPLEMENTAR}

Os espectros uni (1D) e bidimensionais (2D) de ${ }^{1} \mathrm{H}$ RMN e ${ }^{13} \mathrm{C}$ RMN das azinas sintetizadas, bem como os IV e imagem das preparações fotoprotetoras estão disponíveis em http://quimicanova.sbq. org.br, na forma de arquivo PDF, com acesso livre.

\section{AGRADECIMENTOS}

Ao CNPq e CAPES pelo aporte financeiro através de bolsas e a UNIVASF pelo apoio com reagentes e equipamentos.

\section{REFERÊNCIAS}

1. http://www2.inca.gov.br/wps/wcm/connect/tiposdecancer/site/home/ pele_melanoma, acessada em fevereiro 2019; http://www2.inca.gov. br/wps/wcm/connect/tiposdecancer/site/home/pele_nao_melanoma, acessada em fevereiro 2019; http://www1.inca.gov.br/vigilancia/, acessada em fevereiro 2019.

2. Francis, S. O.; Mahlberg, M. J.; Johnson, K. R.; Ming, M. E.; Dellavalle, R. P.; J. Am. Acad. Dermatol. 2006, 55, 849.

3. http://bvsms.saude.gov.br/bvs/saudelegis/anvisa/2012/ rdc0030_01_06_2012.pdf, acessada em fevereiro 2019.

4. http://sbd.tempsite.ws/capele/gestao/caracteristicas_fotoprotecao. asp $? \mathrm{uf}=\mathrm{NULL} \& \mathrm{cidade}=\mathrm{NULL} \& \operatorname{servico}=\mathrm{NULL} \&$ campanha $=16$, acessada em fevereiro 2019.

5. https://www.google.com/url?sa=t\&rct=j\&q=\&esrc $=\mathrm{s} \& \mathrm{~s}$ o u r ce $=$ w e b \& c d $=1 \& \mathrm{c}$ a d $=\mathrm{rj}$ a $\&$ u a c $\mathrm{t}=8 \& \mathrm{ve} d=2$ ahUKEwjQhr2G7MLgAhWXH7kGHbjLD80QFjAAegQIAxA B\&url=http $\% 3 \mathrm{~A} \% 2 \mathrm{~F} \% 2 \mathrm{Fportal}$.anvisa.gov.br\%2Fresultado-debusca\%3Fp_p_id\%3D101\%26p_p_lifecycle\%3D0\%26p_p_ state\%3Dmaximized\%26p_p_mode\%3Dview\%26p_p_col_ id\%3Dcolumn-1\%26p_p_col_count\%3D1\%26_101_struts_ action\%3D\%252Fasset_publisher\%252Fview_content\%26_101_ assetEntryId\%3D2868557\%26_101_type\%3Dcontent\%26_101_
groupId\%3D219201\%26_101_urlTitle\%3Dfiltro-solar-medicamentoou-cosmetico\%26inheritRedirect\%3Dtrue\&usg=AOvVaw3cdwhk Tx-euOUGsJdyq4ms, acessada em fevereiro 2019.

6. Kurteva, V. B.; Simeonov, S. P.; Stoilova-Disheva, M., 2011; Pharmacol. Pharm. 2011, 2, 1; International Union of Pure and Applied Chemistry (IUPAC); Compendium of Chemical Terminology, $2^{\text {nd }}$ ed., Blackwell Scientific Publications: Oxford, 1997.

7. Ramakrishnan, A.; Chourasiya, S. S.; Bharatam, P. V.; RSC Adv. 2015, 5, 55938 .

8. Ha, S-T.; Koh, T-M.; Lee, S-L.; Yeap, G-Y.; Lin, H-C.; Ong, S-T.; Liq. Cryst. 2010, 37, 547 .

9. Sauro, V. A.; Workentin, M. S.; J. Org. Chem. 2001, 66, 831; Zuman, P.; Ludvik, J.; Tetrahedron Lett. 2000, 41, 7851.

10. Lewis, M.; Glaser, R.; J. Org. Chem. 2002, 67, 1441.

11. Safari, J.; Gandomi-Ravandi, S.; RSC Adv. 2014, 4, 46224.

12. Loghmani-Khouzani, H.; Sadeghi, M. M. M.; Safari, J.; Sabzi-Fini. O.; J. Sci., Islamic Repub. Iran 2001, 12, 233; Shang, Z.; Reiner, J.; Chang, J.; Zhao, K.; Tetrahedron Lett. 2005, 46, 2701.

13. Gul, H. I.; Gul, M.; Vepsälainen, J.; Erciyas, E.; Hänninen, O.; Biol. Pharm. Bull. 2003, 26, 631.

14. Jayabharathi, J.; Thanikachalam, V.; Thangamani, A.; Padmavathy, M.; Med. Chem. Res. 2007, 16, 266.

15. Khan, K. M.; Jamil, W.; Ambreen, N.; Taha, M.; Perveen, S.; Morales, G. A.; Ultrason. Sonochem. 2014, 21, 1200.

16. Zarnegar, Z.; Alizadeh, R.; Ahmadzadeh, M.; Safari, J.; J. Mol. Struct. 2017, 1144,58 .

17. Lee, B.; Kang, S. H.; Kang, D.; Lee, K. H.; Cho, J.; Nam, W.; Han, O. H.; Hur, N. H.; Chem. Commun. 2011, 47, 11219.

18. Zhang, M.; Shang, Z-R.; Li, X-T.; Zhang, J-N.; Wang, Y.; Li, K.; Li, Y-Y.; Zhang, Z-H.; Synth. Commun. 2017, 47, 178.

19. Safari, J.; Gandomi-Ravandi, S.; Shariat, S.; J. Iran. Chem. Soc. 2016, $13,1499$.

20. Kaboudin, B.; Alipour, S.; Heteroat. Chem. 2012, 23, 304.

21. Eshghi, H.; Hosseini, M.; J. Chin. Chem. Soc. 2008, 55, 636.

22. Safari, J.; Gandomi-Ravandi, S.; Synth. Commun. 2011, 41, 645.

23. Lenardão, E. J.; Freitag, R. A.; Dabdoub, M. J.; Batista, A. C. F.; Silveira, C. C.; Quim. Nova 2003, 26, 123.

24. Achar, T. K.; Bose, A.; Mal, P.; Beilstein J. Org. Chem. 2017, 13, 1907.

25. Howard, J. L.; Cao, Q.; Browne, D. L.; Chem. Sci. 2018, 9, 3080.

26. Beyer, M. K.; Clausen-Schaumann, H.; Chem. Rev. 2005, 105, 2921.

27. Takacs, L.; Chem. Soc. Rev. 2013, 42, 7649.

28. Mansur, J. S.; Breder, M. N. R.; Mansur, M. C. A.; Azulay, R. D.; An. Bras. Dermatol. 1986, 61, 121

29. Oliveira-Junior, R. G.; Souza, R. G.; Guimarães, A. L.; Oliveira, A. P.; Araújo, C. S.; Silva, J. C.; Pacheco, A. G. M.; Lima-Saraiva, S. R. G.; Rolim, L. A.; Rolim Neto, P. J.; Castro, R. N.; Almeida, J. R. G. S.; Afr. J. Pharm. Pharmacol. 2015, 9, 576.

30. Araújo, C. R. M.; Santos, V. L. A.; Guimarães, D. G.; Gonsalves, A. A.; Journal of Analytical \& Pharmaceutical Research 2018, 7, 222.

31. Pavia, D. L.; Lampman, G. M.; Kriz, G. S.; Vyvyan, J. R.; Introdução à espectroscopia, $4^{\text {th }}$ ed., Cengage Learning: São Paulo, 2010.

32. Rebolledo, A. P.; Lima, G. M.; Gambi, L. N.; Speziali, N. L.; Maia, D. F.; Pinheiro, C. B.; Ardisson, J. D.; Cortés; M. E.; Beraldo, H.; Appl. Organometal. Chem. 2003, 17, 945.

33. Flor, J.; Davolos, M. R.; Correa, M. A.; Quim. Nova 2007, 30, 153.

34. Nascimento, L. F.; Santos, E. P.; Aguiar, A. P.; Rev. Virtual Quim. 2014, 6,190 .

35. Santos, E. P.; Freitas, Z. M.; Souza, K. R.; Garcia, S.; Int. J. Cosmet. Sci. 1999, 21,1 